\title{
Turbulence generation and decay in the Taylor-Couette system due to an abrupt stoppage
}

\author{
H. Singh and A. Prigent $\dagger$ \\ LOMC, UMR CNRS 6294, Université du Havre 53, Rue Prony, CS 80540 76058, Le Havre, France
}

(Received xx; revised xx; accepted xx)

This study presents an innovative approach towards the generation and decay of turbulence in the Taylor-Couette system. The outer cylinder was brought to an abrupt stoppage that generated turbulence in the system, which was initially in the laminar flow regime. Two complementary experimental approaches, namely visualizations and stereo-particle image velocimetry (PIV) measurements, were used to better understand the presented phenomenon for only external cylinder rotation. A moving time average technique was developed due to the continuous change in the length scales throughout the generation and decay process. The different stages of the generation and decay of turbulence were described and characterized through dynamic quantities such as the kinetic energy. This new approach towards the generation and decay of turbulence in the Taylor-Couette flow should help significantly in future endeavours.

\section{Key words:}

\section{Introduction}

Turbulence, a naturally occurring phenomenon, remains a mystery for the scientific community due to its highly non-linear, and spatially and temporalyl fluctuating nature together with a wide range of scales. It occurs when energy is provided to the system, for example through rotation, temperature gradient and flow velocity. The higher this input of energy is, the stronger the turbulence will be. At the same time, the turbulence dissipates kinetic energy (Verschoof et al. 2016). A major part of this decay of turbulence has been researched through the lens of homogeneous isotropic turbulence (HIT) (Taylor 1935; de Karman \& Howarth 1938; Kolmogorov 1941; Batchelor et al. 1948a,b; Saffman 1967; Sreenivasan 1984; George 1992; Lohse 1994; Skrbek \& Stalp 2000; Eyink \& Thomson 2000; Hurst \& Vassilicos 2007; Davidson 2011; Meldi et al. 2011; Thormann \& Maneveau 2014; McComb et al. 2014; Valente \& Vassilicos 2015; Sinhuber et al. 2015; Vassilicos 2015; Antonia et al. 2017; McComb \& Fairhurst 2018). How this decay happens is still a subject of vast research due to non-consensus on its major aspects: (1) Do initial conditions influence the decay? (George 1992; Biferale et al. 2003; Thormann \& Maneveau 2014; Sinhuber et al. 2015); (2) Is the decay self similar? (Antonia et al. 2017; Batchelor et al. 1948a; George 1992; Eyink \& Thomson 2000; ?; Verschoof et al. 2016; Ostilla-Mónico et al. 2017; Sinhuber et al. 2015); (3) Is the decay dependent on the Reynolds number?(George 1992; de Karman \& Howarth 1938; Saffman 1967; Lohse 1994; Sinhuber et al. 2015; McComb et al. 2014); And (4) what is the rate of decay (Saffman 1967; George 1992; Sinhuber et al. 2015; Thormann \& Maneveau 2014)?

The importance of clearly understanding how turbulence develops and decays is not just fundamental but also of significant practical interest. The famous two equation $k-\epsilon$ turbulence

$\dagger$ Email address for correspondence: arnaud.prigent@univ-lehavre.fr 
model, $d \epsilon / d t=-C_{\epsilon 2} \epsilon^{2} / k$, used in simulations is based on the homogenous isotropic turbulence assumption, and the constant, $C_{\epsilon 2}$, is estimated from the rate of decay Thormann \& Maneveau (2014). At the same time, this $k-\epsilon$ turbulence model is known for excessively damping turbulence scales and under-predicts its dissipation (Singh et al. 2011). A better understanding of fundamental aspects of turbulence will definitely help in the improvement of the existing turbulence models or in creating a new model altogether.

It is important to remember that in the early nineteenth century a lot of assumptions were considered to simplify the theoretical calculations, one of which is HIT. However, turbulence is chaotic in nature and therefore, generally, neither homogeneous nor isotropic. Consequently, in recent years, a new approach is being developed towards wall-bounded turbulence decay to observe this chaotic behaviour either numerically (Touil et al. 2002; Schneider \& Farge 2008; Ostilla-Mónico et al. 2017; Schikarski \& Avila 2017) or experimentally (Peixinho \& Mullin 2006; Verschoof et al. 2016). All of these studies have been conducted in different types of geometries: pipe flow (Peixinho \& Mullin 2006), two plates (Touil et al. 2002), two-dimensional structures (Schneider \& Farge 2008), T-mixer (Schikarski \& Avila 2017), and the Taylor-Couette (TC) system (Verschoof et al. 2016; Ostilla-Mónico et al. 2017). Each geometrical system has its own advantages, but the TC system, in which flow is contained between two concentric cylinders, presents itself as an ideal system because it is a confined and closed system with non-homogeneous and anisotropic turbulence (Huisman et al. 2014). For example, its biggest advantage over the pipe flow is its modest size, which makes it more practical to study decay in a wall-bounded environment both experimentally and numerically.

Verschoof et al. (2016) studied experimentally, using PIV and laser Doppler velocimetry (LDV), the decay in turbulence by stopping the inner cylinder, while the outer one was at rest, and allowing the turbulence to decay freely from the ultimate turbulent regime at a Reynolds number of $10^{6}$. However, they faced a practical problem of stopping the cylinder instantaneously. In their case, it took $12 \mathrm{~s}$ to reach a complete stop and during this time they did not measure the decay. Consequently, the group provided a follow-up numerical study (Ostilla-Mónico et al. 2017) in which the inner cylinder was brought to a halt abruptly, but at the much smaller Reynolds number of $3.5 \times 10^{4}$. Also described by Batchelor et al. (1948a,b), they (Ostilla-Mónico et al. 2017) observed three distinctly different stages of decay: initial, intermediate and final. Both concluded that, during the initial stage, the energy decayed through large eddies (Batchelor et al. 1948a) or rolls (Ostilla-Mónico et al. 2017) and the final decay stage remained purely viscous. The intermediate stage represented continual change in turbulent motion with inertial terms strong enough to maintain the transfer of energy from the large eddies (Batchelor et al. 1948a).

In this manuscript, a slightly different phenomenon is presented. Instead of starting from a turbulent flow and studying its decay, we started from a laminar flow. The rotation of the outer cylinder was fixed in order to obtain a laminar flow and after five minutes the cylinder was stopped abruptly (with details provided in the methodology). Generation of turbulence was observed shortly after the stoppage, which decayed freely afterwards due to absence of any further driving force given to the system. As a result, this experimental work differs significantly from the experimental work of Verschoof et al. (2016) according to the following points: i) the flow was in a laminar state before the instantaneous stoppage in comparison with a turbulent state; ii) the outer cylinder was in motion before being stopped in comparison with the inner cylinder; iii) it was stopped abruptly in comparison with a gradual stoppage; and iv) both the turbulence generation and free decay are observed in comparison with free turbulence decay.

It shares some similarities with the works on impulsive spin-down in a cylinder (Euteneuer \& Karlsruhe 1972; Neitzel \& Davis 1981; Neitzel 1982; Mathis \& Neitzel 1985; Savas 1992; Kim \& Choi 2004, 2006; Kim et al. 2008; Kaiser et al. 2020) or Couette flow decay in the TC system (Kohuth \& Neitzel 1988; Neitzel 1982; Tillmann 1967). While the geometry of the first case differs, it can be seen as a particular case of the TC system (with radius ratio, $\eta=0$ ) and Kohuth 
\& Neitzel (1988) have shown that the inner cylinder of the TC system has no influence on the onset of the instability at large Reynolds number. Thus, what happens at the very beginning when the cylinder is stopped can be compared to what happens in the TC system when the outer cylinder is stopped. The whole process of the generation and decay of turbulence has been described in the direct numerical simulations by Kaiser et al. (2020). They considered an infinitely long cylinder in solid-body rotation (SBR) and impulsively stopped for a wide range of Reynolds numbers. They have identified five stages according to their flow structure and their underlying mechanisms of kinetic energy dissipation. Initially, the laminar boundary layer undergoes a primary centrifugal instability, which causes the formation of coherent Taylor rolls. The flow then becomes turbulent, once the Taylor rolls are destabilized by secondary instabilities. Within the turbulent stage, they distinguished two phases. In the first one, the SBR core is still intact and turbulence is sustained. They described the mean velocity profile by the superposition of a near-wall region, a retracting SBR core and an intermediate region of constant angular momentum. In the second turbulent phase, the SBR core breaks down, turbulence starts to decay exponentially and the kinetic energy of the mean flow decays logarithmically. Finally, the flow becomes laminar again and the velocity profile of the analytical solution for purely laminar decay is recovered.

In the TC flow, this problem has been addressed as a way to study instabilities of an unsteady circular Couette flow. As far as we know, very few authors (Kohuth \& Neitzel 1988; Neitzel 1982; Tillmann 1967) have worked on this subject and all considered the case where the cylinders were initially in SBR and then the outer one was stopped while the inner one remained in motion. In this configuration, no final decay was observed as energy was still injected to the flow through the rotation of the inner cylinder. The final state was either the Taylor vortex flow, turbulence or the circular Couette flow but it was not reached after decay from turbulence. Experimental works did not provide velocity measurements. Here, the generation and decay of turbulence are studied experimentally using two complementary approaches: visualizations with Kalliroscope and stereo-PIV measurements to capture all the three velocity components. The paper is organized as follows. In section 2, the methodology is presented and contains the description of the experimental set-up and the averaging technique used to obtain the results described and discussed in section 3. Finally, the conclusion gives a summary and outlook in section 4.

\section{Methodology}

\subsection{Apparatus}

The TC system consists of two cylinders with inner and outer radii of $r_{i}=0.04 \mathrm{~m}$ and $r_{o}=0.05$ $\mathrm{m}$, respectively, with a radius ratio, $\eta=r_{i} / r_{o}$, of 0.8 and gap width, $d=r_{i}-r_{o}$, of $0.01 \mathrm{~m}$. The maximum height of the working fluid, $L$, is $0.45 \mathrm{~m}$ with aspect ratio $\Gamma=L / b=45$. Degassed water was used as the working fluid at a constant temperature of $20^{\circ} \mathrm{C}$ maintained in the water circulation in the inner cylinder and the envelope surrounding the outer cylinder. The inner cylinder (IC) is made of aluminum having black anodized wall to avoid reflection when working with laser. The outer cylinder is made of Plexiglas to provide optical access over the entire gap width axially. The system also contains a bottom and top ring, attached to the outer cylinder at a distance of $0.5 \mathrm{~mm}$ from the inner cylinder. Only outer cylinder rotation was considered in these experiments. In each run, the flow conditions were laminar before stopping the cylinder abruptly using the emergency stop button. For the emergency stop, the deceleration of system was measured to be constant and $\approx 2.8 \mathrm{~m} / \mathrm{s}^{2}$. With this deceleration, it always took less than a half of a rotation of the outer cylinder to come to a complete halt, irrespective of the initial rotational velocity. For the largest Reynolds number, the outer cylinder rotational velocity drops from 34.9 to $0 \mathrm{rad} / \mathrm{s}$ in 0.0017 viscous time units $\left(t v / d^{2}\right)$ or $\approx 29.7$ inertial time units $\left(t r_{o} \omega_{o} / d\right)$. 
It corresponds to 0.43 revolution. If this stoppage time is compared with the experimental time, it takes $\approx 0.4 \%$ of time for the stoppage in comparison with the decay of velocity from its maximum to reach 0 . In this article both the viscous and inertial time units have been used. As suggested by Neitzel \& Davis (1981), various times scales can be used to present the results. Among them, the inertial time units can be used for comparison within the same geometry; while, he viscous time units are preferred to compare with different geometries.

In order to visualize flow structures, the degassed water was mixed with $1 \%$ Kalliroscope ST-1000. A $r-z$ plane was illuminated with a class 4 Ray Power 2000 continuous laser with a maximum output of $4 \mathrm{~W}$ at $532 \mathrm{~nm}$ wavelength. This $r-z$ plane was captured with a Phantom $\mathrm{M}$ series lab140 CMOS camera of 4MP resolution having a maximum of $800 \mathrm{fps}$ speed using an objective of $105 \mathrm{~mm}$. The Dantec Dynamics studio version 6.0 was used to capture and export the images which were then treated with Matlab R2018a software to obtain the presented results. A maximum of 2000 to 10000 single frame images were captured depending upon the acquisition frequency of 50 and $200 \mathrm{~Hz}$ (at higher Reynolds number), respectively.

The Stereo-PIV measurements were also done in the $r-z$ plane through the transparent outer cylinder using two cameras and objectives identical to the one used for visualizations. The cameras were synchronized with a Nd:YAG medium Class 4 Litron laser LPY 50-200 having maximum output of $200 \mathrm{~mJ}$, wavelength of $532 \mathrm{~nm}$, and a maximum frequency of acquisition of $200 \mathrm{~Hz}$. However, in order to capture at least 35 seconds of the process, $50 \mathrm{~Hz}$ and $100 \mathrm{~Hz}$ (at higher Reynolds numbers) acquisition frequency were used to capture around 2500 and 3800 double image pairs, respectively. The fluid was seeded with fluorescent Rhodamine particles of average size between $5-20 \mu \mathrm{m}$. The calibration of both cameras was done with the pinhole method of the image modeling fit methodology using a 3D calibration target manufactured by Dantec dynamics for stereo-PIV measurements for this specific TC system. The captured images were analyzed initially with the Dantec Dynamics studio version 6.4 to compute the vector fields using the adaptive PIV method having the maximum interrogation area (IA) size of $32 \times 32$ grid, minimum IA size of $8 \times 8$ grid and grid step size of $4 \times 4$. In this method, the IA size decreases automatically from the maximum towards the minimum IA's specified provided the particle density is high enough, and the grid step size controls the vector spacing. Afterwards, the calibrations of both cameras were selected along with the vector fields of both cameras to create the stereo-PIV vector fields which were imported as *.txt files and treated with Matlab R2018a software to present the results.

\subsection{Temporal and spatial averaging}

The averaged data were calculated using both space and time averaging. The spatial averaging was performed along the axial direction. As all scales change with time during the decay process, the traditional time averaging process, when a certain amount of data is averaged over time to obtain statistically converged data, was not feasible. Instead, a moving time average is considered here, in which the time averaging takes places during the whole decay process at each individual time-step by considering a certain amount of data before and after that point. $5 \mathrm{fd} / u_{\theta}$ is chosen as the criteria to estimate the number of data points before and after each time-step, such that $t^{\prime}=\left(i-5 f d / u_{\theta}\right):\left(i+5 f d / u_{\theta}\right)$, where $u_{\theta}$ and $f$ represent azimuthal velocity component and frequency of acquisition, respectively. $d / u_{\theta}$ was chosen as the time scale because the evolution of length scales is directly related to the azimuthal velocity component; whereas the factor 5 was chosen to ascertain that there will be a minimum of 10 images to do the averaging process at the start of decay when the $d / u_{\theta}$ is the smallest. The factor influences the number of data points to conduct the mobile average. With an increase in the number of data points for averaging, the temporal dynamics get subdued but without impacting the global behavior or its convergence, as can be observed in Fig. 4 where the factor 5 is compared with a factor 15. This combination of averaging process appeared to be sufficient to obtain converged data. The convergence was mainly 


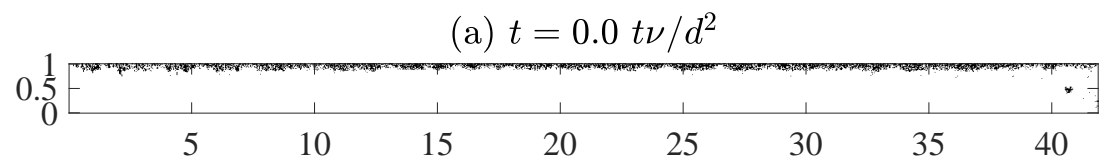

(b) $t=0.005 t \nu / d^{2}$

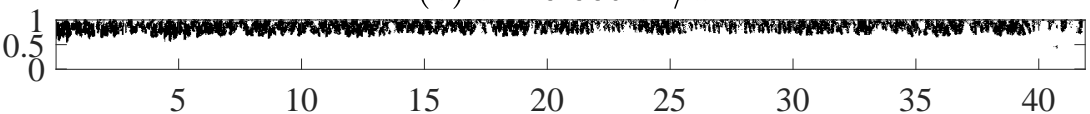

(c) $t=0.01 t \nu / d^{2}$

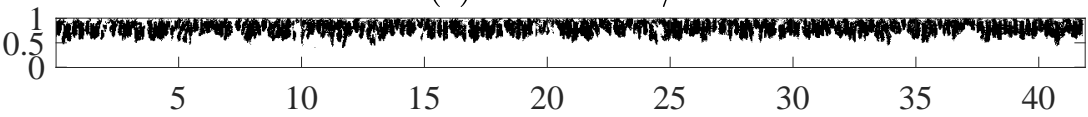

(d) $t=0.017 t \nu / d^{2}$

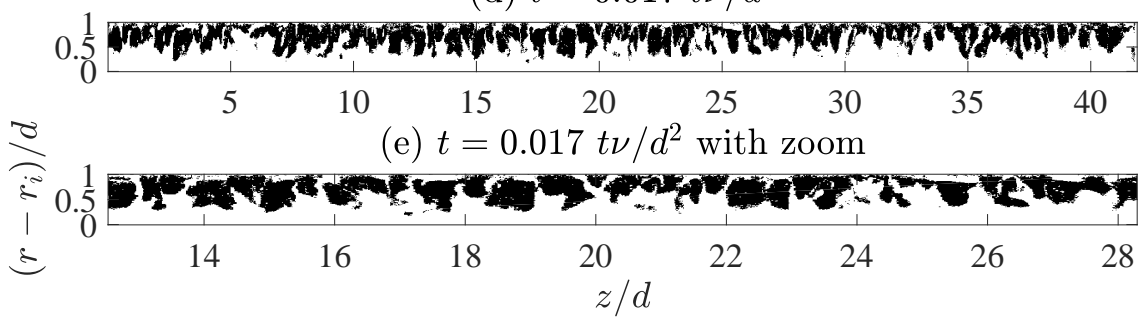

Figure 1: Binarized instantaneous images presenting growth of vortical structures at different times-steps for the $R e_{o}=1700$ over the whole axial height (z) of the TC system.

The $\mathrm{x}$ and $\mathrm{y}$ labels are the same for all sub-figures, as in (e).

achieved by the spatial averaging, as also observed by Kaiser et al. (2020) in their numerical work. We did not perform ensemble average of multiple repetitions for the same $R e_{o}$, but we checked that two independent repetitions have the same behavior, as shown in Fig. 4a.

\section{Results \& Discussion}

\subsection{Visualizations}

When the outer cylinder is stopped abruptly from a velocity greater than a given value, small vortices appear near the outer wall. Then, these vortices grow towards the inner cylinder and are rapidly destabilized. Finally, as no further energy is delivered to the flow, the vortex pattern decays. The whole process is presented for $R e_{o}=\omega_{o} r_{o} d / v=1700$ in a movie available as online supplementary material. Fig. 1 displays different time-steps using binarized instantaneous images over the whole axial height (z) of the TC system at $R e_{o}=1700$. The instantaneous images were binarized, after being normalized by a mean image, using a Matlab function - imbinarize with default criteria for sensitivity of 0.5 . The growth of the vortical structures can be observed from the moment they appear until they fill the whole gap-width, which takes $\approx 0.017$ viscous time units.

Taking the light intensity along a vertical line at mid gap in each acquired image normalized with a mean image, we have constructed space-time diagrams. Fig. 2 presents such space-time diagrams giving the variation in light intensity reflected by the Kalliroscope particles for three different outer Reynolds numbers $R e_{o}=870,1700$ and 17000 . They show the temporal evolution of the generation and decay of vortices and eventually turbulence. On the space-time diagrams, 

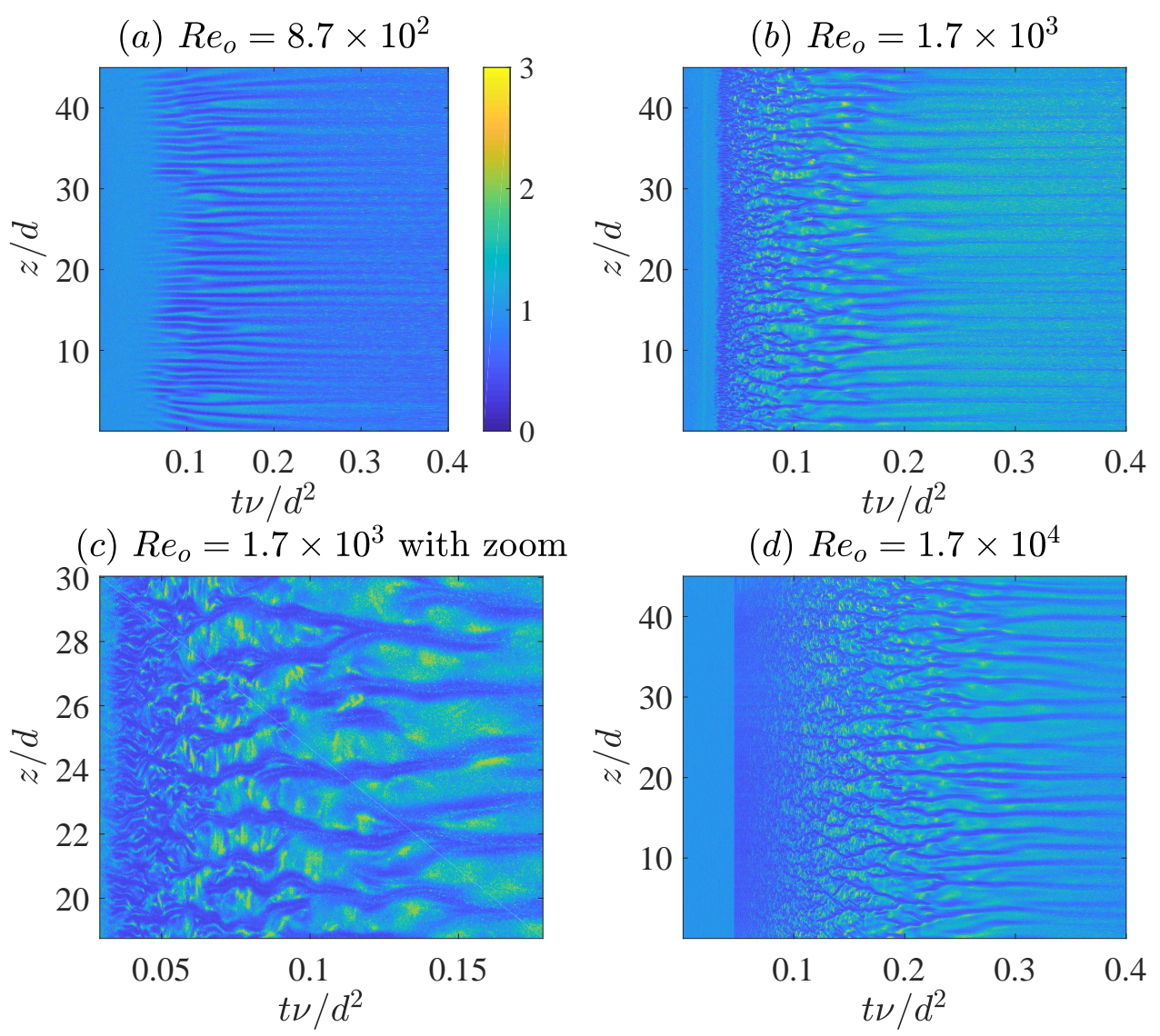

Figure 2: Space-time diagram of the light intensity taken along z near the outer-cylinder for increasing outer Reynolds number with a stationary inner cylinder.

bright areas correspond to areas where Kalliroscope flakes move in the azimuthal direction whereas dark areas correspond to inwards or outwards motion in the radial direction

The minimum $R e_{o}$ at which vortex formation and its decay is observed in the whole axial length is 870 . Vortices appear after the stoppage, merge and fade. However, for this $R e_{o}$ value, even if the merging of the vortices induces some defects the flow is not turbulent (see Fig. 2a). When increasing $R e_{o}$, vortices still appear but rapidly undergo reorganization as they grow. We consider the formation of this disordered flow as the setting in of turbulence (see Fig. 2 b, c and d). The higher the initial outer Reynolds number, the faster the stoppage generates turbulence throughout the axial length and the longer it lasts. It can also be seen on this figure that the size of the structures increases with time. They persist for a very long time and their persistence time increases with $R e_{o}$. At $R e_{o}=1.7 \times 10^{4}$, they are still visible at $t v / d^{2}=0.4$.

We have estimated the size and growth of the vortices after they appear near the outer cylinder. This estimation was performed from the image obtained after binarization of the axially averaged images. We used the same binarization process as mentioned earlier. The initial vortex size $\left\langle\delta_{i}\right\rangle_{z}$ corresponds to the size the vortical structure when it first appears. This is presented in Fig. 3a as a function of the outer Reynolds number $R e_{o}$. It decreases with $R e_{o}$ and follows a power law which is well fitted by $a \times R e_{O}^{-2 / 3}$ as proposed by Kim \& Choi (2004) for the critical wavelength 
(a)

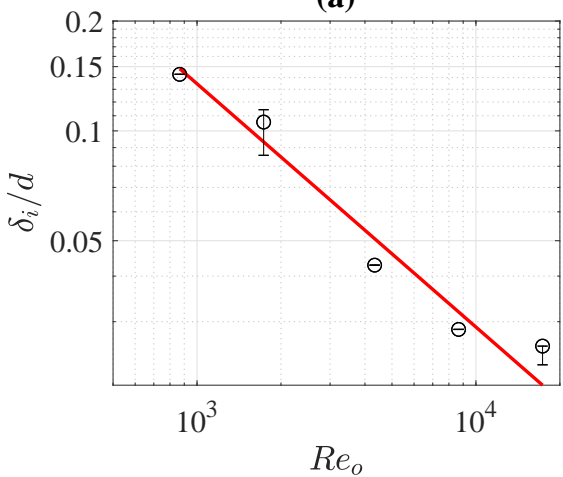

(c)

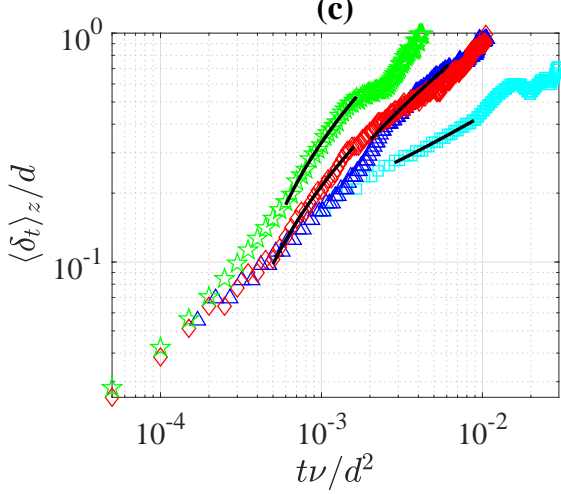

(b)

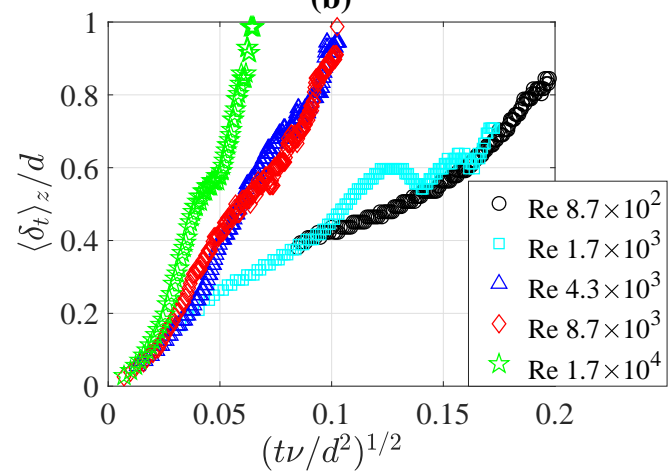

(d)

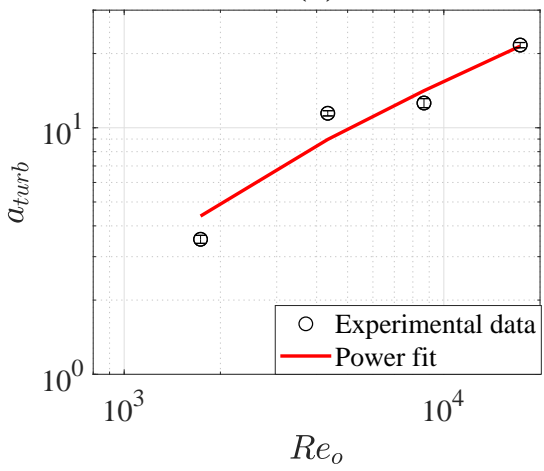

Figure 3: Initial size of the vortex (a), their growth in plot (b) and logarithmic scale (c), and proportionality constant as a function of $\operatorname{Re}_{o}(\mathrm{~d})$. The legend in part (a) is similar to that in (d), and the one in (b) represents (c) as well. In sub-figure (c) symbol represents experimental data and line of the black colour represent fitted curve for the respective $R e_{o}$. The goodness of the fit, $R^{2}$, varied between 0.97 and 0.99 for all power laws. In (d), all coefficients were with a certainty of $95 \%$ confidence bounds, where the error bar represents the upper and lower bounds for each value of $a_{t u r b}$.

of Taylor-Görtler vortices generated in an impulsively stopped rotating cylinder. Moreover, the best power law fit gives $n=-0.65$ which is very close to the expected $-2 / 3$ exponent.

Fig. 3b presents $\left\langle\delta_{t}\right\rangle_{z}$, the size of the vortical structures as a function of $\sqrt{t v / d^{2}}$ for different $R e_{o}$. This estimation was also obtained after binarization of the axially averaged images, but using an adaptive method with a senstivity of 0.6. In viscous time units, the time required by the vortices to fill up the gap width decreases with each increment in the $R e_{o}$. In a consistent way w.r.t. Fig. 3a , the starting point for $\left\langle\delta_{t}\right\rangle_{z}$ decreases with increasing $R e_{o}$. Different growth phases can be distinguished for each $R e_{o}$. The initial growth phase is similar for all $R e_{o}>870$ with a mean growth rate of approximately 4.7. The following growth phases also followed a power law, as such it was pertinent to use a logarithmic scale to present it in Fig. 3c. In this case, the $R e_{o}=870$ was not plotted because it was not turbulent, as mentioned earlier. The curves intersect each other as the $\left\langle\delta_{t}\right\rangle_{z}$ grows with $t v / d^{2}$ but differently for each $R e_{o}$. We found that they can all be well approximated by a power law fit with a same exponent of $1 / 2$ but each with different proportionality constant $a_{t u r b}$, as can be observed in Fig. $3 \mathrm{c}$. Other exponents than $1 / 2$ do not give better results for the fit. 

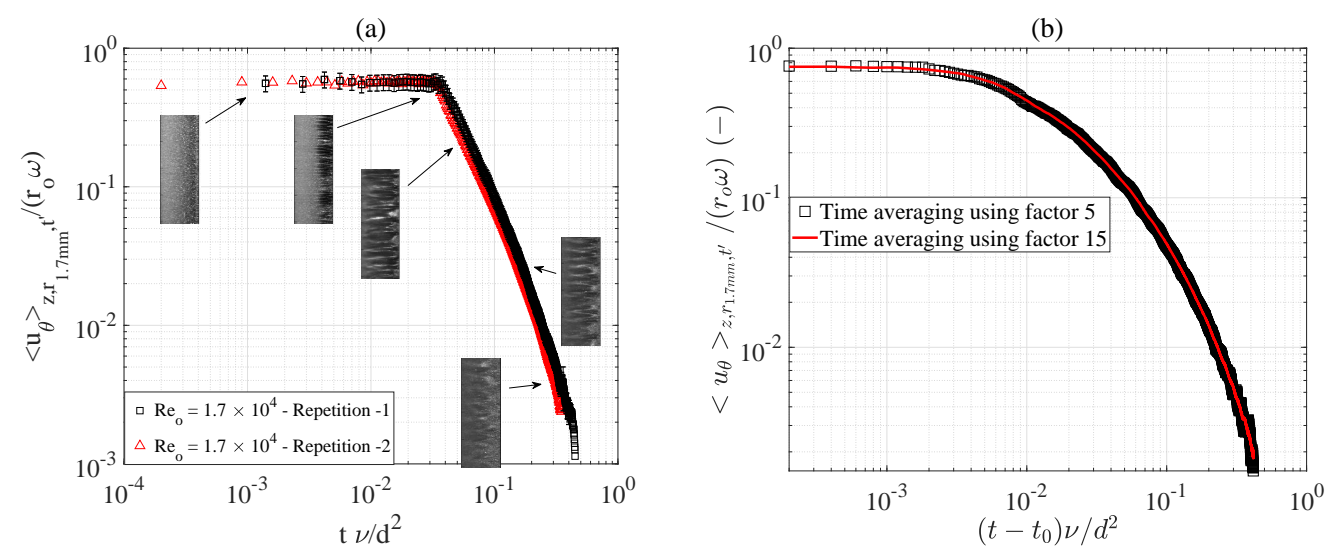

Figure 4: Decay of azimuthal velocity with time at $R e_{o}=17300$. It is averaged axially, radially around the center of gap-width over $2.7 \mathrm{~mm}$, and at each instant of time with $t^{\prime}=\left(i-5 f d / u_{\theta}\right):\left(i+5 f d / u_{\theta}\right)$. In part (a) snapshots of instantaneous visualizations are also presented to show the evolution of the decay of the velocity for two different sets of data: repetition 1 and 2, respectively. In part (b) the data set 1 was used to average with the different factors of 5 and 15 . The $t_{0}$ represents the time at which the stop occurs.

Our results are in very good agreement with the results of Kaiser et al. (2020) and Euteneuer \& Karlsruhe (1972) whose unstable zone grows as the square-root of time. Following the idea that the vortices appear in the unstable zone as the interpenetrating spirals do in the unstable zone between the inner cylinder and the nodal surface (the cylindrical surface where $u_{\theta}=0$ ) in counter-rotating Taylor-Couette flow, we can consider that $\left\langle\delta_{t}\right\rangle_{z}$ approximately corresponds to the size of the unstable zone of the flow. Kaiser et al. (2020) also observed multiple stages of $\sqrt{v t}$ growth, with each one separated by fast growth transition. In another similarity with the results of Kaiser et al. (2020) and Euteneuer \& Karlsruhe (1972), we have found that the coefficient $a_{t u r b}$ increases with $R e_{o}$, as presented in Fig. 3d. We obtained a power law with an exponent of $1 / 2$ and a proportionality constant of 0.18 . Our power law exponent differs from the power law exponent of Kaiser et al. (2020), who obtained a value of 1/3. However, Kaiser et al. (2020) presented the coefficient $a_{t u r b}$ as a ratio of $a_{\text {turb }} / a_{\text {lam }}$, where $a_{\text {lam }}$ is the proportionality factor for the growth of the laminar boundary layer thickness for their phase one and $a_{t u r b}$ corresponds to the phase three.

\subsection{Mean azimuthal velocity decay}

Fig. $4 \mathrm{a}$ and $\mathrm{b}$ presents the evolution of the mean azimuthal velocity with respect to time. The azimuthal velocity was averaged axially over $100 \mathrm{~mm}$ in the mid-height region, radially over 1.7 $\mathrm{mm}$ in the center of gap region and at each instant of time with $t^{\prime}=\left(i-5 f d / u_{\theta}\right):\left(i+5 f d / u_{\theta}\right)$. To reduce the number of data points, the data at every $5^{\text {th }}$ instance is plotted for Fig. 4a. The instantaneous visualization snapshots presented alongside the velocity decay demonstrate the evolution of the generation and decay process. The visualization and PIV velocity estimations were conducted separately and do not necessarily represent the actual time-specific evolution but rather the probability of its observation in that time zone. In Fig. $4 \mathrm{~b}$, the time origin $t_{0}$ is fixed at the time $t v / d^{2}=0.034$ of Fig. $4 \mathrm{a}$

In Fig. 4a it seems that the mean azimuthal velocity's rate of decay follows a power law of the type $y=a x^{n}$. However, when only the decay region is observed in Fig. 4b, it can be seen that this decay doesn't follow a single power law, as also observed by Ostilla-Mónico et al. (2017). Multiple decay regions can be observed in the Fig. 4b. We have identified three phases for the 

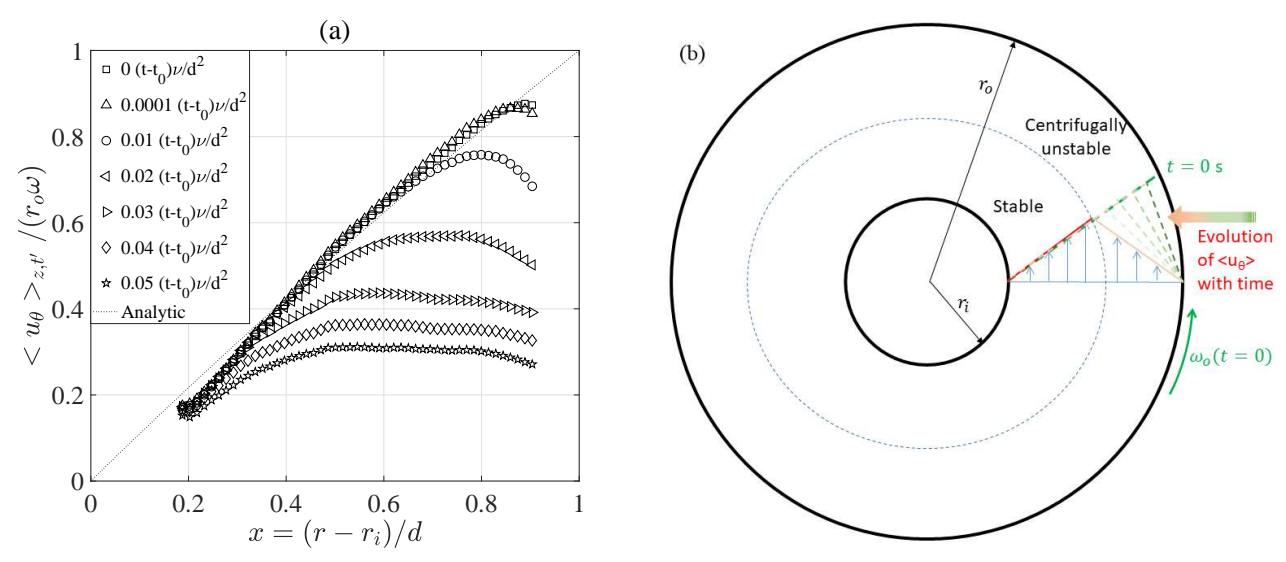

Figure 5: Radial decay process observed experimentally at $R e_{O}=1700$ (a) and its theoretical explanation (b). In (a) mean azimuthal velocity is averaged axially and at each instant of time with $t^{\prime}=\left(i-5 f d / u_{\theta}\right):\left(i+5 f d / u_{\theta}\right)$. The dotted line represents the analytic laminar profile. The $t_{0}$ represents the time at which the stop occurs.

whole turbulence generation and decay process. These three phases can be better understood with the observation of the variation of the turbulent kinetic energy in the following sub-section.

An important aspect to be observed is the free decay of the azimuthal velocity profile as a function of time (Fig. 5). Fig. 5a gives the azimuthal velocity profile at different time steps of the decay period as shown in Fig.4b. The azimuthal velocity is averaged axially and at each instant of time using the moving average method mentioned earlier. A selected few time-steps were chosen to present the decay of the turbulence. As for the visualizations, $R_{o}=1700$ is chosen to present the decay process of the mean azimuthal radial profile over the first 0.05 viscous time units. At $R e_{o}=17300$, this decay process was much faster and took just 0.01 viscous time units for the radial profile to reach below $40 \%$ of the maximum azimuthal velocity component. The two first profiles, for $t=0$ and $0.0001\left(t-t_{0}\right) v / d^{2}$, are very close to the analytical Couette profile, which is represented by the analytical profile presented as the dotted line. Then, as the outer cylinder is stopped, the next profiles remain close to the Couette flow in the first half of the gap, reach a maximum value, termed $u_{\theta \max }$, and decrease towards zero. Our measurements did not allow us to observe the decrease toward zero near the outer cylinder. These profiles are similar to the ones observed in spin-down experiments (Kim et al. 2008; Kaiser et al. 2020). The shape and structure of the azimuthal velocity profiles demonstrate that the unstable region lies near the outer cylinder for the current configuration, which can also be confirmed in the Fig. 1.

Fig.5 was constructed after estimating the actual point of stoppage, which could be observed clearly for the visualization experiments, but is not known as well for the stereo-PIV measurements. Based on the Fig.4, we can deduce the actual time-frame at which the decay starts; though, the question remains if the point at which decay starts is same at which the stop occurs. However, this decay process ascertains that at the instance of stoppage the azimuthal velocity component at the outer cylinder wall will fall towards zero and cause this decay observed in Fig.5. As a result, it is fair to assume that the point of decay is the point of stoppage as well.

As demonstrated in the Fig. 5b, as soon as the cylinder is stopped, the velocity of the outer cylinder wall becomes zero. The wall velocity at the inner cylinder is already zero (because inner cylinder is stationary), but the velocity in the gap-width remains non-zero. This creates a centrifugally unstable region viz a viz Rayleigh's criteria (Rayleigh 1917), which states that if the Rayleigh's discriminant is positive an instability may develop. Hence, as soon as the outer cylinder 
wall velocity becomes zero, the Rayleigh's discriminant becomes positive in the region between the position of $u_{\theta \max }$ and the outer cylinder creating the possibility of a centrifugal instability. At the first stage of the instability, the unstable region and the resulting vortices are located in the immediate vicinity of the outer cylinder. As stated by Neitzel (1982) for experiments of Couette flow decay, the inner cylinder has probably no influence on the instability at this stage. These vortical structures grow along with the thickness of the unstable region. Therefore, the pattern requires continuous reorganization that leads to its irregularity and the development of turbulence in the system depending upon the Reynolds number (Fig. 2). These structures propagate towards the inner cylinder (Fig 1), with the growth rate depending on the Reynolds number as explained earlier. Similar observations were reported by Euteneuer \& Karlsruhe (1972) and Neitzel \& Davis (1981).

Fig. 6 presents the azimuthal velocity profile corresponding to the instance at which decay starts at different $R e_{o}$. It was averaged in a similar way as in the case for Fig. 5. It demonstrates the size of the unstable zone which could be governing the appearance of the vortices. It is known that the size of the zone between the nodal surface and inner cylinder walls plays a critical role in the localisation and size of vortices induced by the destabilization of the counter-rotating Couette flow (Esser \& Grossmann 1996). Similarly, the zone between the inflection point, observable in Fig. 6, and the outer cylinder wall must determine the appearance and the size of the vortical structures.

\subsection{Kinetic energy decay}

Fig. 7 and 8 demonstrates the decay of the three forms of the kinetic energy (KE): mean, turbulent and wind. The presented data is averaged axially, radially near the center of gap for Fig. 7 and near the inner and outer cylinder for Fig. 8, and at each instant of time with $t^{\prime}=\left(i-5 \mathrm{fd} / u_{\theta}\right)$ : $\left(i+5 f d / u_{\theta}\right)$. Fig. $7 \mathrm{a}, \mathrm{b}$ and $\mathrm{c}$ presents a comparison for the three types of KE among different $R e_{o}$; while, Fig. $7 \mathrm{~d}$ and 8 depicts these three types of KE only for the $R e_{o}=1.7 \times 10^{4}$. All the temporal scales in Fig. 7 and 8 are dimensioneled using the dynamic time-scale $r_{o} \omega / d$ to facilitate comparison between different $R e_{o}$. Additionally, all the temporal profiles for these kinetic energies start from the moment stop occurs as discussed for Fig. 4. The mean KE was estimated from the mean velocity flow components: $k_{\text {mean }}=1 / 2\left(\left\langle u_{r}\right\rangle_{t^{\prime}, z}^{2}+\left\langle u_{\theta}\right\rangle_{t^{\prime}, z}^{2}+\left\langle u_{z}\right\rangle_{t^{\prime}, z}^{2}\right)$, where $r$ and $z$ represent the radial and axial coordinates. The turbulent $\mathrm{KE}$ is estimated from the fluctuating radial, azimuthal and axial components of the velocity as: $k_{t u r b}=1 / 2\left(\left(\left\langle u_{r}^{\prime 2}\right\rangle_{t^{\prime}, z}+\left(\left\langle u_{\theta}^{\prime 2}\right\rangle_{t^{\prime}, z}+\left(\left\langle u_{z}^{\prime 2}\right\rangle_{t^{\prime}, z}\right)\right.\right.\right.$, where $u_{r, \theta, z}^{\prime}=u_{r, \theta, z}-\left\langle u_{r, \theta, z}\right\rangle_{t^{\prime}, z}$. And finally, the wind $\mathrm{KE}$ was composed of only the radial and axial fluctuating components of the velocity: $k_{\text {wind }}=1 / 2\left(\left\langle u_{r}^{\prime 2}\right\rangle_{t^{\prime}, z}+\left\langle u_{z}^{\prime 2}\right\rangle_{t^{\prime}, z}\right)$. It can be noticed that the general behavior of the three kinetic energies is the same for all $R e_{o}$. As the Reynolds number fixed the initial conditions of the decay process, it means that this initial condition has no impact on the general behavior of the decay. Temporal re-scaling with the $r_{o} \omega / d$ term makes the decay profile longer for increasing $R e_{o}$. Considering the similar behavior for all $R e_{o}$, we compare directly the three kinetic energies in the Fig. $7 \mathrm{~d}$ for the largest $R e_{o}$.

The mean KE follows the mean azimuthal velocity profile and decays continuously with time. The turbulent and wind KE profiles show different phases for this turbulence generation and decay process. When the outer cylinder is rotating, its vibration generates experimental noise which gives the initial value for the turbulence and wind kinetic energies. As the outer cylinder is stopped, the turbulence sets in and these kinetic energies starts to grow until they reach their maximum value. This generation of turbulence is considered as the phase I of this generation and decay process. As also observed in Fig. 2, this initial phase is marked by the generation of small vortical structures resulting of the centrifugal instability. These small scale structures induce radial and axial components of the velocity, resulting in an increase in the wind KE as observed in Fig. 7d. At the center of the gap, the time at which the maximum is reached is $49 t r_{o} \omega / d$; 


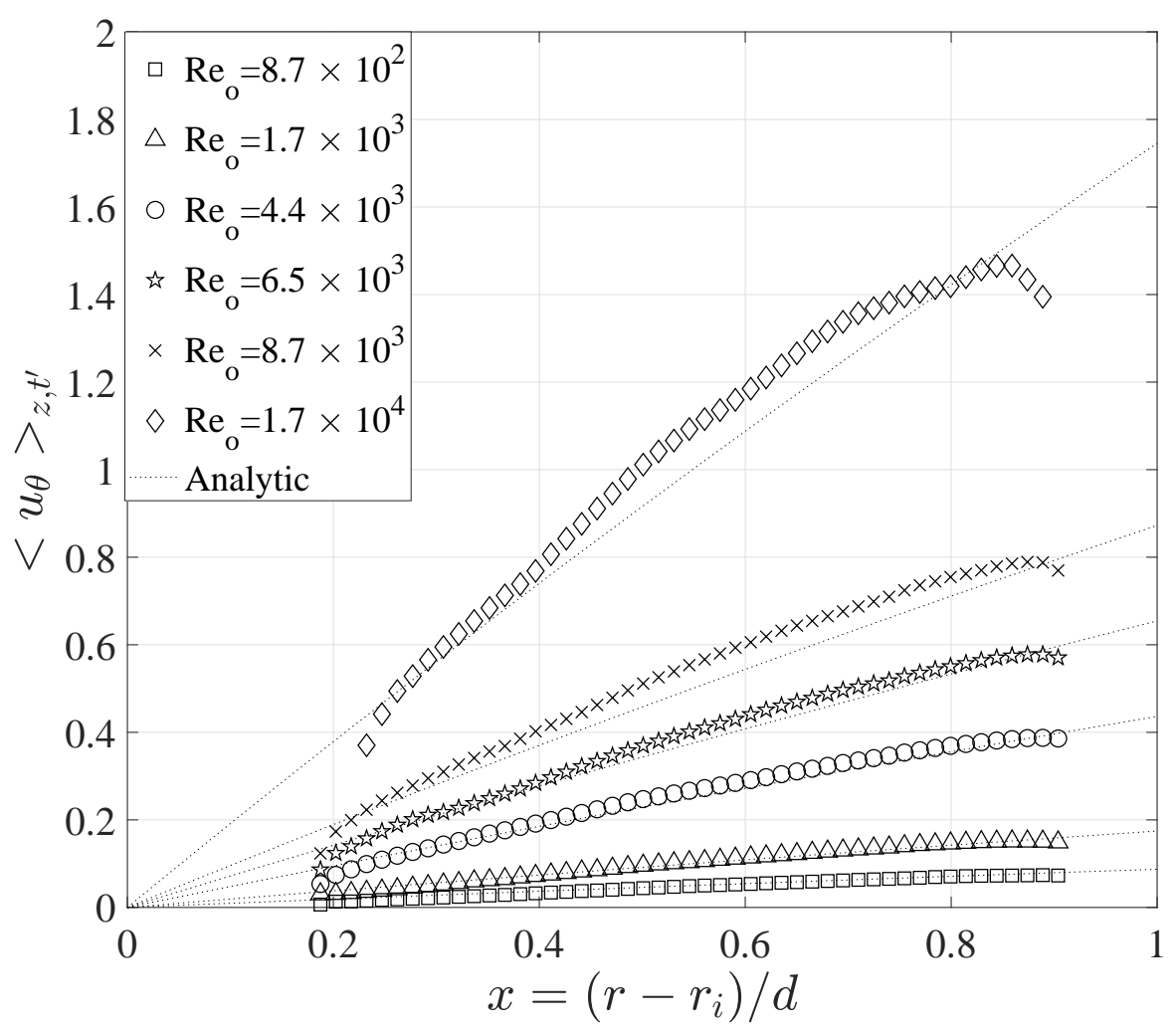

Figure 6: Azimuthal velocity at the instance at which decay starts for different $R e_{o}$. It is averaged axially and at each instant of time with $t^{\prime}=\left(i-5 f d / u_{\theta}\right):\left(i+5 f d / u_{\theta}\right)$. The vertical red line points towards the inflection point observable at the presented Reynolds number.

whereas, it is 100 near the inner cylinder, as can be seen in Fig. 8. Near the outer cylinder, there is a small initial decrease followed by a small plateau between $t r_{o} \omega / d$ of 31 and 45 . At this location the velocity is closed to its maximal value and the velocity fluctuations as well. When the cylinder is stopped, the fluctuations slightly decrease with the velocity but the emergence of the structures maintain their high level. Then, as the velocity further decreases, the decay process starts and the wind and turbulence kinetic energies decrease from their maximum values at the center and near the inner cylinder; and from the plateau near the outer cylinder. This occurs at different times for each positions along the gap. This time difference between the different starting points of the decay comes from the propagation of the structures from the outer cylinder towards the inner cylinder as they grow (Fig 1). Kaiser et al. (2020) observed similar phenomenon in their Phase-II which they termed as "emergence of Taylor rolls and laminar-to-turbulent transition". Evidently, this initial phase is not present if the flow is already turbulent before the stop occurs, as in the results of Verschoof et al. (2016) and Ostilla-Mónico et al. (2017). There is no generation of turbulence occurring in this case which could have lead to an increase in the turbulent KE.

Once the maximum is reached, the decay of the turbulent and wind KE starts. This decay phase is named phase II on Fig. 7d. We found that the wind KE appears to follow power law with the power exponent of -1.36 . This falls between the HIT based theoretical predictions of $-6 / 5$, and $-10 / 7$ for Saffman (1967), and Kolmogorov (1941) theories, respectively. Phase II is 

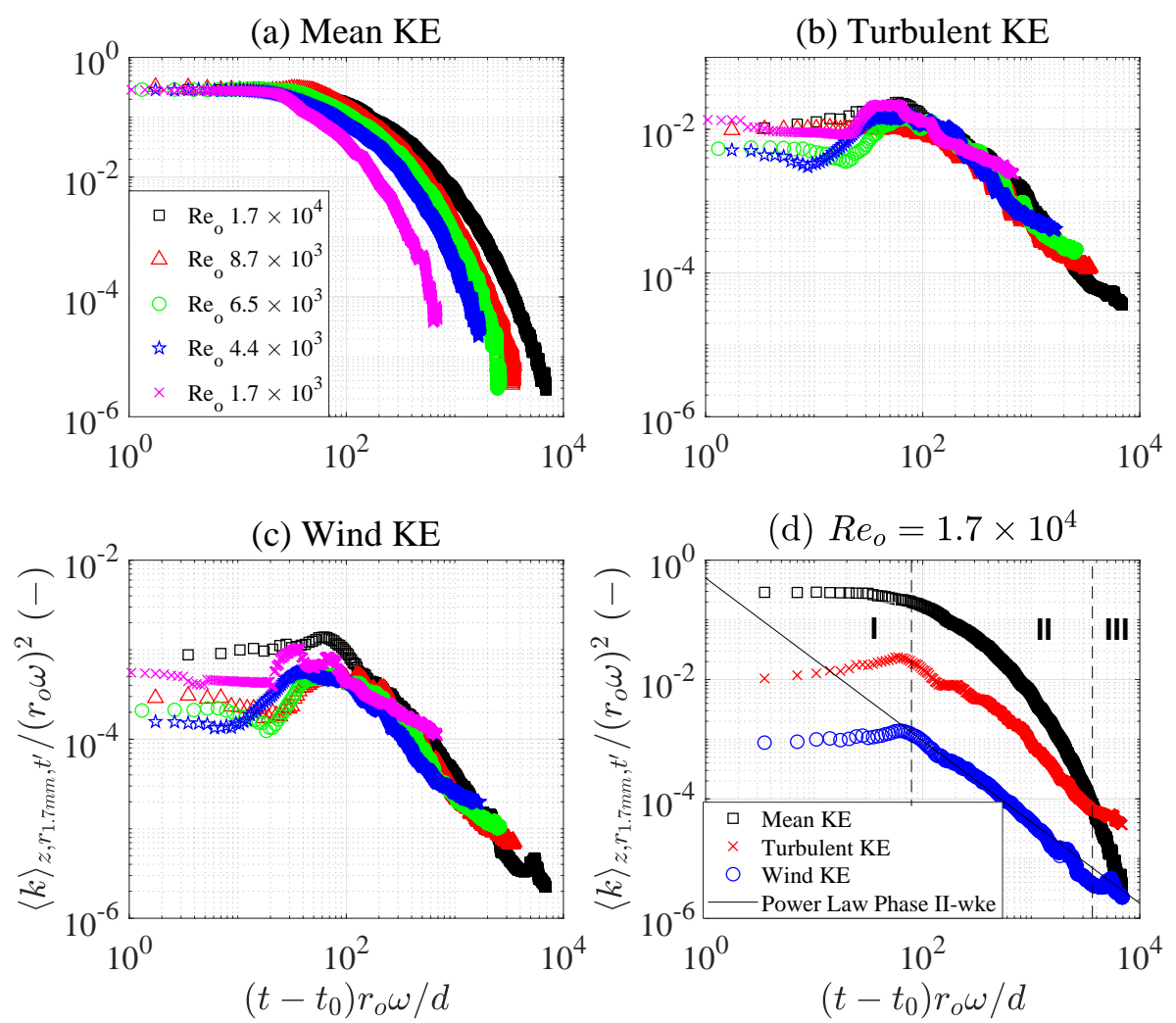

Figure 7: Temporal evolution of the dimensionless mean (a), turbulent (b) and wind KE (c) at different Reynolds numbers, and these three together for $R e_{o}=1.7 \times 10^{4}$ (d). These are averaged axially, radially around the center of gap over $1.7 \mathrm{~mm}$ and and at each instant of time with $t^{\prime}=\left(i-5 f d / u_{\theta}\right):\left(i+5 f d / u_{\theta}\right)$. The $\mathrm{x}$ and y labels for sub-figures $\mathrm{a}$ and $\mathrm{b}$ is same as that in $\mathrm{c}$ and $\mathrm{d}$. Also, the legend in sub-figures $\mathrm{b}$ and $\mathrm{c}$ is same as that in $\mathrm{a}$. The $t_{0}$ represents the time at which the stop occurs.

defined by the merging of smaller scale structures towards larger scales together with their fading. This process is repeated until the final phase (phase III in Fig. 7d), which is dominated by the viscous diffusion (Fig. 2). The merging of structures leads to the reorganization of the energy and the length scales continuously. During this process of reorganization, multiple plateaus can be remarked upon, for example the biggest one around $150 t r_{o} \omega / d$ of decay in Fig. $7 \mathrm{~d}$. These plateaus are visible around the center and near the inner cylinder but not near the outer cylinder (Fig. 7d and 8). The fusion of the structures at certain instances sustains the KE causing these plateaus, this process can also be observed in Ostilla-Mónico et al. (2017). Once this fusion stops, the decay process enters the final phase during which the large scale structures decay under the influence of viscous diffusion.

Towards the end of this final decay phase, the turbulent KE was higher in magnitude compared to the mean KE; even though, at this stage the flow cannot be considered as turbulent. This occurred whatever the measurement position was. At this stage, we observe large vortical structures having an axial periodicity and propagating tangentially as axisymmetric rolls which decay with time, as can be observed in the Fig. 2. Following the idea of Hussain \& Reynolds (1970), these vortical structures with their axial periodicity can be associated to a wave component in the velocity 


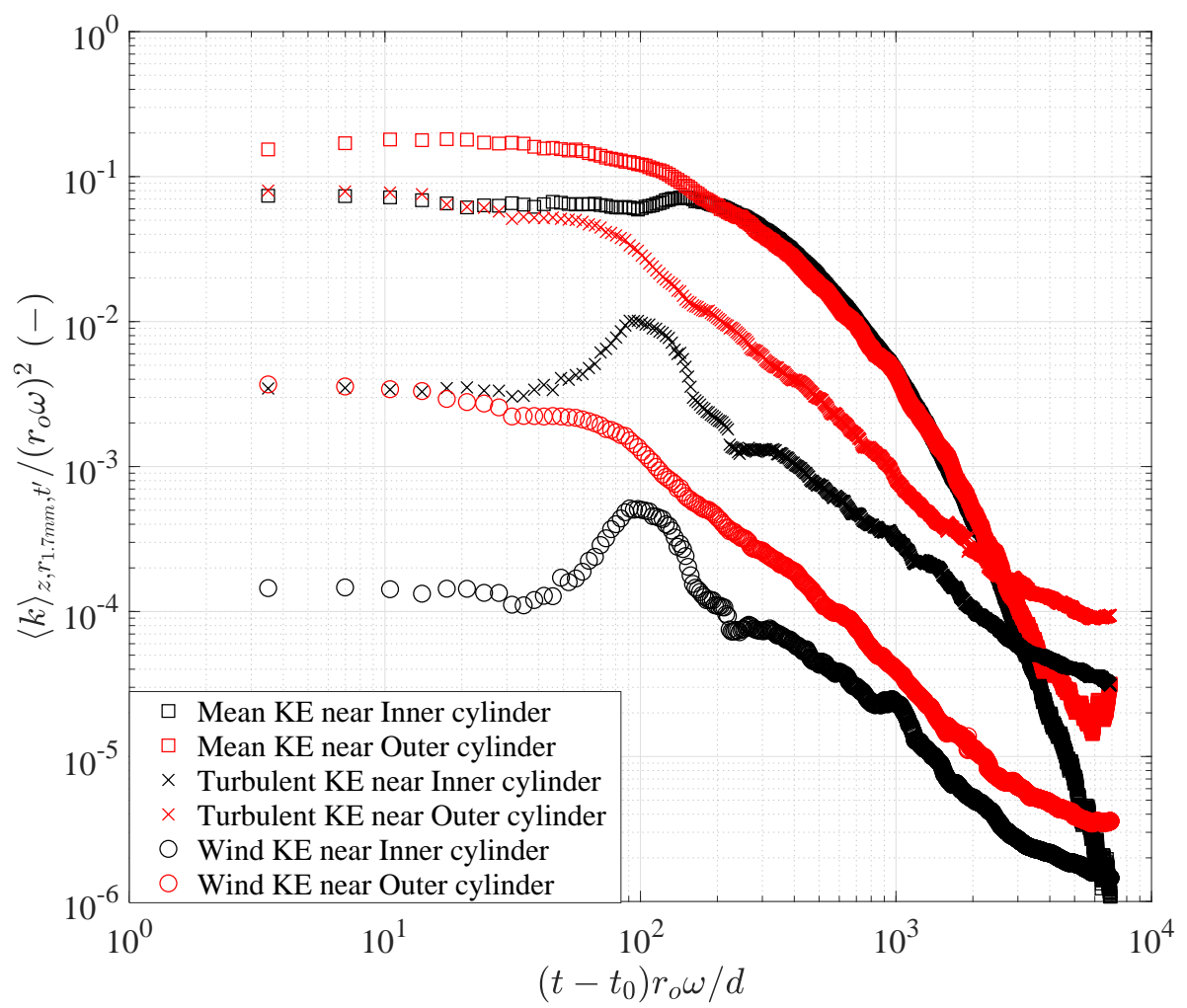

Figure 8: Temporal evolution of the dimensionless mean, turbulent and wind $\mathrm{KE}$ at $R e_{o}=17300$ when radially averaged near inner (black markers) and outer (red markers) cylinder. These are averaged axially, radially around the inner cylinder (black markers) and outer cylinder (red markers) over $1.7 \mathrm{~mm}$ and and at each instant of time with $t^{\prime}=\left(i-5 f d / u_{\theta}\right):\left(i+5 f d / u_{\theta}\right)$. The $t_{0}$ represents the time at which the stop occurs.

decomposition process. In this process $u=\langle u\rangle+u^{\prime}$, where $u^{\prime}=u_{\text {wave }}+u_{\text {turbulence }}$. In our case, there is a non-separation of the wave component from the fluctuating velocity component (Hussain \& Reynolds 1970) which forms the turbulent KE. As such, the turbulent KE represents both the wave component due to the vortical structures and the fluctuating component. At the end of the decay process, the $u^{\prime}$ is mainly composed of the wave component.

\subsection{Energy dissipation rate and length scales}

Fig. 9a presents the production of KE and the mean, turbulent and total KE dissipation rates estimated using the 7 out of 12 gradients using the cylindrical coordinates (Pope 2000). The direct estimation of the energy dissipation of the mean KE, turbulent KE and total KE can be estimated from the Equations 3.1, 3.2 and 3.3, respectively. The current PIV measurements were stereoPIV which provides access to the three velocity components but only two set of coordinates. As such out of the total 12 components for the direct estimation of the dissipation of the mean, turbulent and total KE, only seven were estimated here because other five components required the azimuthal coordinate which was not accessible, as shown in Equations 3.4, 3.5 and 3.6. Nonetheless, Singh (2016) have shown that the missing five components related to the azimuthal coordinate had negligible contribution in the estimation of the mean KE dissipation rate with 

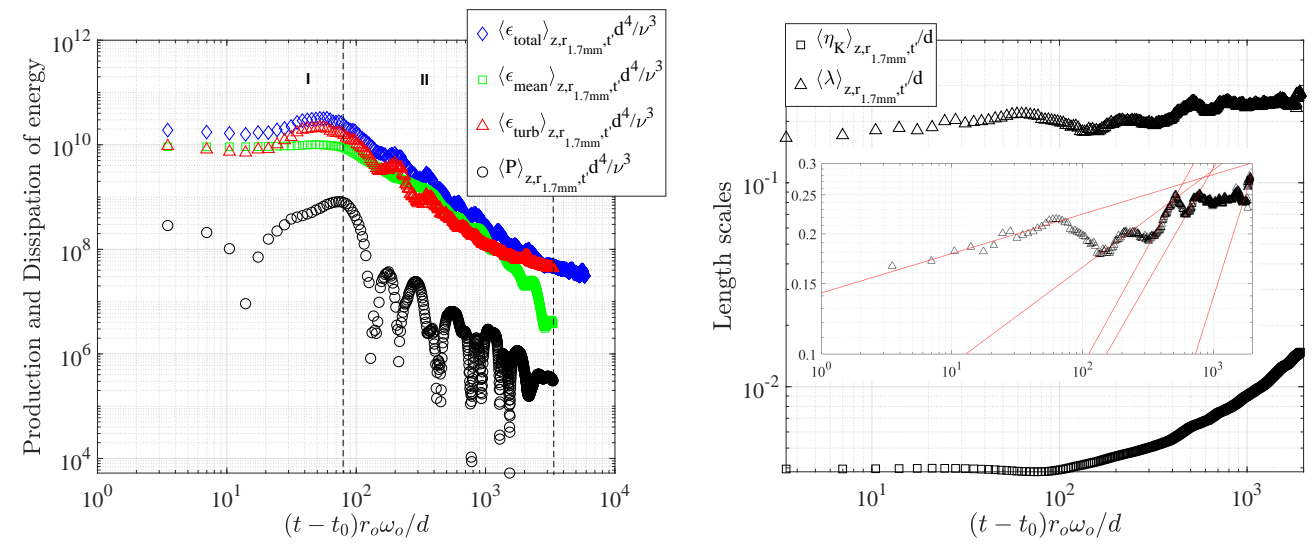

Figure 9: Temporal evolution of the production and dissipation of energy and two length scales at $R e_{O}=17300$. The insert in b presents the multiple power laws observed in the Taylor microscale. These are averaged axially, radially around the center of gap-width over $1.7 \mathrm{~mm}$ and and at each instant of time with $t^{\prime}=\left(i-5 f d / u_{\theta}\right):\left(i+5 f d / u_{\theta}\right)$. The $t_{0}$ represents the time at which the stop occurs.

more than $90 \%$ contribution coming from the gradients of the radial coordinate. The symmetry of the roll structures that appear in this case is similar to that of the turbulent Taylor vortex flow. Moreover, these missing five components can be estimated using the Taylor's hypothesis, which would give $\partial \theta=u_{\theta} \partial t / r$. A brief analysis of these components showed less than $0.01 \%$ contribution towards the mean energy dissipation rate estimation. Consequently, the prediction based on the seven components was maintained. These gradients were estimated using the $2^{\text {nd }}$ order central differencing approximation of the first derivative (Singh 2016).

Based on the estimation of the dissipation rate, the Taylor microscale, $\langle\lambda\rangle=\sqrt{15 v\left\langle u_{\theta}^{\prime 2}\right\rangle /\left\langle\epsilon_{\text {mean }}\right\rangle}$, and Kolmogorov scale, $\left\langle\eta_{K}\right\rangle=\left(v^{3} /\left\langle\epsilon_{\text {mean }}\right\rangle\right)^{(1 / 4)}$ were also estimated. These two length scales are presented in Fig. 9b. On the other hand, the production of KE was estimated as: $P=-\left\langle u_{\theta}^{\prime} u_{r}^{\prime}\right\rangle\left(d\left\langle u_{\theta}\right\rangle / d r-u_{\theta} / r\right)$. Fig. 9a and b shows only the data corresponding to the phase I and II, of the KE, except for the profile of the total energy dissipation. Only the phase I and II were chosen because the viscous decay phase III was practically diffusive in nature and contained insignificant fluctuating content, as can be observed with the temporal evolution of the turbulent and wind KE (Fig. 7).

$$
\begin{aligned}
\epsilon_{\text {mean }_{\text {all }}} & =v\left\{2\left(\left(\frac{\partial \overline{U_{r}}}{\partial r}\right)^{2}+\left(\frac{1}{r} \frac{\partial \overline{U_{\theta}}}{\partial \theta}+\frac{\overline{U_{r}}}{r}\right)^{2}+\left(\frac{\partial \overline{U_{z}}}{\partial z}\right)^{2}\right)+\left(r \frac{\partial \overline{\left(U_{\theta} / r\right)}}{\partial r}\right)^{2}\right. \\
& +\left(\frac{1}{r} \frac{\partial \overline{U_{r}}}{\partial \theta}\right)^{2}+\left(\frac{1}{r} \frac{\partial \overline{U_{z}}}{\partial \theta}\right)^{2}+\left(\frac{\partial \overline{U_{z}}}{\partial r}\right)^{2}+\left(\frac{\partial \overline{U_{r}}}{\partial z}\right)^{2}+\left(\frac{\partial \overline{U_{\theta}}}{\partial z}\right)^{2} \\
& \left.+2\left(\frac{\partial \overline{\left(U_{\theta} / r\right)}}{\partial r} \cdot \frac{\partial \overline{U_{r}}}{\partial \theta}+\frac{1}{r} \frac{\partial \overline{U_{\theta}}}{\partial z} \cdot \frac{\partial \overline{U_{z}}}{\partial \theta}+\frac{\partial \overline{U_{r}}}{\partial z} \cdot \frac{\partial \overline{U_{z}}}{\partial r}\right)\right\}
\end{aligned}
$$




$$
\begin{aligned}
& \epsilon_{\text {turb }} \text { all }=v\left\{2\left(\left(\frac{\partial u_{r}^{\prime}}{\partial r}\right)^{2}+\left(\frac{1}{r} \frac{\partial u_{\theta}^{\prime}}{\partial \theta}+\frac{u_{r}^{\prime}}{r}\right)^{2}+\left(\frac{\partial u_{z}^{\prime}}{\partial z}\right)^{2}\right)+\left(r \frac{\partial\left(u_{\theta}^{\prime} / r\right)}{\partial r}\right)^{2}\right. \\
& +\left(\frac{1}{r} \frac{\partial u_{r}^{\prime}}{\partial \theta}\right)^{2}+\left(\frac{1}{r} \frac{\partial u_{z}^{\prime}}{\partial \theta}\right)^{2}+\left(\frac{\partial u_{z}^{\prime}}{\partial r}\right)^{2}+\left(\frac{\partial u_{r}^{\prime}}{\partial z}\right)^{2}+\left(\frac{\partial u_{\theta}^{\prime}}{\partial z}\right)^{2} \\
& \left.+2\left(\frac{\partial\left(u_{\theta}^{\prime} / r\right)}{\partial r} \cdot \frac{\partial u_{r}^{\prime}}{\partial \theta}+\frac{1}{r} \frac{\partial u_{\theta}^{\prime}}{\partial z} \cdot \frac{\partial u_{z}^{\prime}}{\partial \theta}+\frac{\partial u_{r}^{\prime}}{\partial z} \cdot \frac{\partial u_{z}^{\prime}}{\partial r}\right)\right\} \\
& \epsilon_{\text {total }_{\text {all }}}=v\left\{2\left(\left(\frac{\partial U_{r}}{\partial r}\right)^{2}+\left(\frac{1}{r} \frac{\partial U_{\theta}}{\partial \theta}+\frac{U_{r}}{r}\right)^{2}+\left(\frac{\partial U_{z}}{\partial z}\right)^{2}\right)+\left(r \frac{\partial\left(U_{\theta} / r\right)}{\partial r}\right)^{2}\right. \\
& +\left(\frac{1}{r} \frac{\partial U_{r}}{\partial \theta}\right)^{2}+\left(\frac{1}{r} \frac{\partial U_{z}}{\partial \theta}\right)^{2}+\left(\frac{\partial U_{z}}{\partial r}\right)^{2}+\left(\frac{\partial U_{r}}{\partial z}\right)^{2}+\left(\frac{\partial U_{\theta}}{\partial z}\right)^{2} \\
& \left.+2\left(\frac{\partial\left(U_{\theta} / r\right)}{\partial r} \cdot \frac{\partial U_{r}}{\partial \theta}+\frac{1}{r} \frac{\partial U_{\theta}}{\partial z} \cdot \frac{\partial U_{z}}{\partial \theta}+\frac{\partial U_{r}}{\partial z} \cdot \frac{\partial U_{z}}{\partial r}\right)\right\} \\
& \epsilon_{\text {mean }}=v\left(2\left(\left(\frac{\partial \overline{U_{r}}}{\partial r}\right)^{2}+\left(\frac{\partial \overline{U_{z}}}{\partial z}\right)^{2}\right)+\left(r \frac{\partial \overline{\left(U_{\theta} / r\right)}}{\partial r}\right)^{2}+\left(\frac{\partial \overline{U_{z}}}{\partial r}\right)^{2}+\left(\frac{\partial \overline{U_{r}}}{\partial z}\right)^{2}\right. \\
& \left.+\left(\frac{\partial \overline{U_{\theta}}}{\partial z}\right)^{2}+2 \frac{\partial \overline{U_{r}}}{\partial z} \cdot \frac{\partial \overline{U_{z}}}{\partial r}\right) \\
& \epsilon_{t u r b}=v\left(2\left(\left(\frac{\partial u_{r}^{\prime}}{\partial r}\right)^{2}+\left(\frac{\partial u_{z}^{\prime}}{\partial z}\right)^{2}\right)+\left(r \frac{\partial\left(u_{\theta}^{\prime} / r\right)}{\partial r}\right)^{2}+\left(\frac{\partial u_{z}^{\prime}}{\partial r}\right)^{2}+\left(\frac{\partial u_{r}^{\prime}}{\partial z}\right)^{2}\right. \\
& \left.+\left(\frac{\partial u_{\theta}^{\prime}}{\partial z}\right)^{2}+2 \frac{\partial u_{r}^{\prime}}{\partial z} \cdot \frac{\partial u_{z}^{\prime}}{\partial r}\right) \\
& \epsilon_{\text {total }}=v\left(2\left(\left(\frac{\partial U_{r}}{\partial r}\right)^{2}+\left(\frac{\partial U_{z}}{\partial z}\right)^{2}\right)+\left(r \frac{\partial\left(U_{\theta} / r\right)}{\partial r}\right)^{2}+\left(\frac{\partial U_{z}}{\partial r}\right)^{2}+\left(\frac{\partial U_{r}}{\partial z}\right)^{2}\right. \\
& \left.+\left(\frac{\partial U_{\theta}}{\partial z}\right)^{2}+2 \frac{\partial U_{r}}{\partial z} \cdot \frac{\partial U_{z}}{\partial r}\right)
\end{aligned}
$$

The turbulent KE dissipation rate and the production of KE increased in the phase I (Fig. 9a). This further confirms that the phase I is defined by the generation of KE. The initial (first three points) higher magnitude of the production of $\mathrm{KE}$ and the turbulent $\mathrm{KE}$ dissipation rate is due to the vibration of the outer cylinder while it was rotating before the stop, as mentioned earlier. The phase II starts with the decay of all energy dissipation rates. During this decay process occurs large oscillations of the production of $\mathrm{KE}$ and small oscillations of all the KE dissipation rates. During this phase also occurs the reorganization of the vortices. This induces the oscillations of the turbulent and mean KE dissipation rates but at different instances. The behaviour of the total $\mathrm{KE}$ dissipation rate, which is the sum of the turbulent and mean KE dissipation rates, reflects its two components oscillations. The production of KE represents the transfer of energy between the turbulent and mean components of the flow. Its oscillations correspond to the oscillations of the total KE dissipation rate. Towards the end of Phase II, the turbulent KE dissipation rate was higher 
in magnitude than the mean KE dissipation rate. As explained earlier, the turbulent component, presented herein, consists of both the fluctuating and periodic components. Towards the end of decay phase, the fluctuating component is negligible, so that the turbulent KE mainly consists of the periodic component which is due to axisymmetric vortices. This will continue until these vortices vanish completely. This is the main reason for higher turbulent KE dissipation rate than the mean KE dissipation rate towards the end of decay process. As a consequence, the profile of the total KE dissipation rate is similar to that of the turbulent KE dissipation rate. Verschoof et al. (2016) and Ostilla-Mónico et al. (2017) presented total KE dissipation rate that is more similar to the profile of the mean KE dissipation rate presented here. In the case of Verschoof et al. (2016) and Ostilla-Mónico et al. (2017), there was no generation of turbulence, and only decay was happening from the moment the inner cylinder was brought to a stop.

George (1992) stated that Taylor microscale should be considered as the characteristic length scale, and that it increases as the square root of time, or $\lambda \sim t^{1 / 2}$. An increase, following power law, could be seen multiple times but not constantly in phases I and II Fig. 9b. The power law exponent increases from 0.1 to 1 as a function of time, as can be observed from the insert in Fig. 9b. Moreover, Taylor microscale, which is known to play a dominant role in the energy and momentum transfer, captures well the reorganization of energy. The Kolmogorov scale is constant during the first 100 inertial time units and then its increases monotonically with time. This scale by definition is inversely proportional to the mean $\mathrm{KE}$ dissipation rate, which is clearly visible in its temporal profile. The reorganization of the length scales and the energy are not as visible in the mean KE dissipation rate, and consequently not represented by the Kolmogorov scale. As such, the Taylor microscale better describes the decay of energy, and should be considered as the length scale for the phases I and II in comparison to the Kolmogorov scale, even though the increase as the square root of time was not observed.

\subsection{Self-similarity}

Fig. 10 presents the self-similarity picture for the radial profile azimuthal velocity at $R e_{o}=$ 17300 for the decay phases. Velocity profile was averaged axially and over time, and normalising it with its spatial average. This was formed based on the self-similarity criteria presented by Verschoof et al. (2016). They defined the criteria for self-similarity as that when the azimuthal velocity profiles averaged over $\theta$ are normalized with the spatial mean of azimuthal velocity averaged radially and azimuthally, the profiles overlapped each other. The only difference with their method being that instead of using azimuthal velocity profiles averaged over $\theta$, azimuthal velocity was averaged axially and over time. A complete self-similarity covering the whole decay process was not seen. Instead, profiles under phase II were found to be self-similar among themselves but not much in the final phase. Verschoof et al. (2016) observed self-similarity only in the intermediate region; whereas, Ostilla-Mónico et al. (2017) observed self-similarity in the final decay phase.

Earlier, George (1992) stated different characteristics for a self-preserving solution: $\lambda$ and $R_{\lambda}=u^{\prime} \lambda / v$ as the characteristic length scale and turbulence Reynolds number respectively; energy decay follows a power law; Taylor microscale can be predicted as the square root of time; constants of proportionality and exponent vary w.r.t the initial condition, and finally that the spectrum and spectral functions collapse when plotted. As per characteristics of a self preserving solution defined by George (1992), some similarities could be seen. Most notably, Taylor microscale, $\lambda$, could be considered as the characteristic length scale based on its temporal evolution depiction in the Fig. 9b. Energy decay was observed to be following a power law, but not a single power law that could define the whole decay process due to reorganization of structures and length scales, as can be observed in the Fig. 7 and 8.

On the other hand, it is crucial to observe how the decay will occur at a much larger outer cylinder Reynolds number, of the order of $10^{6}$. If it can be done experimentally it would be much better. 


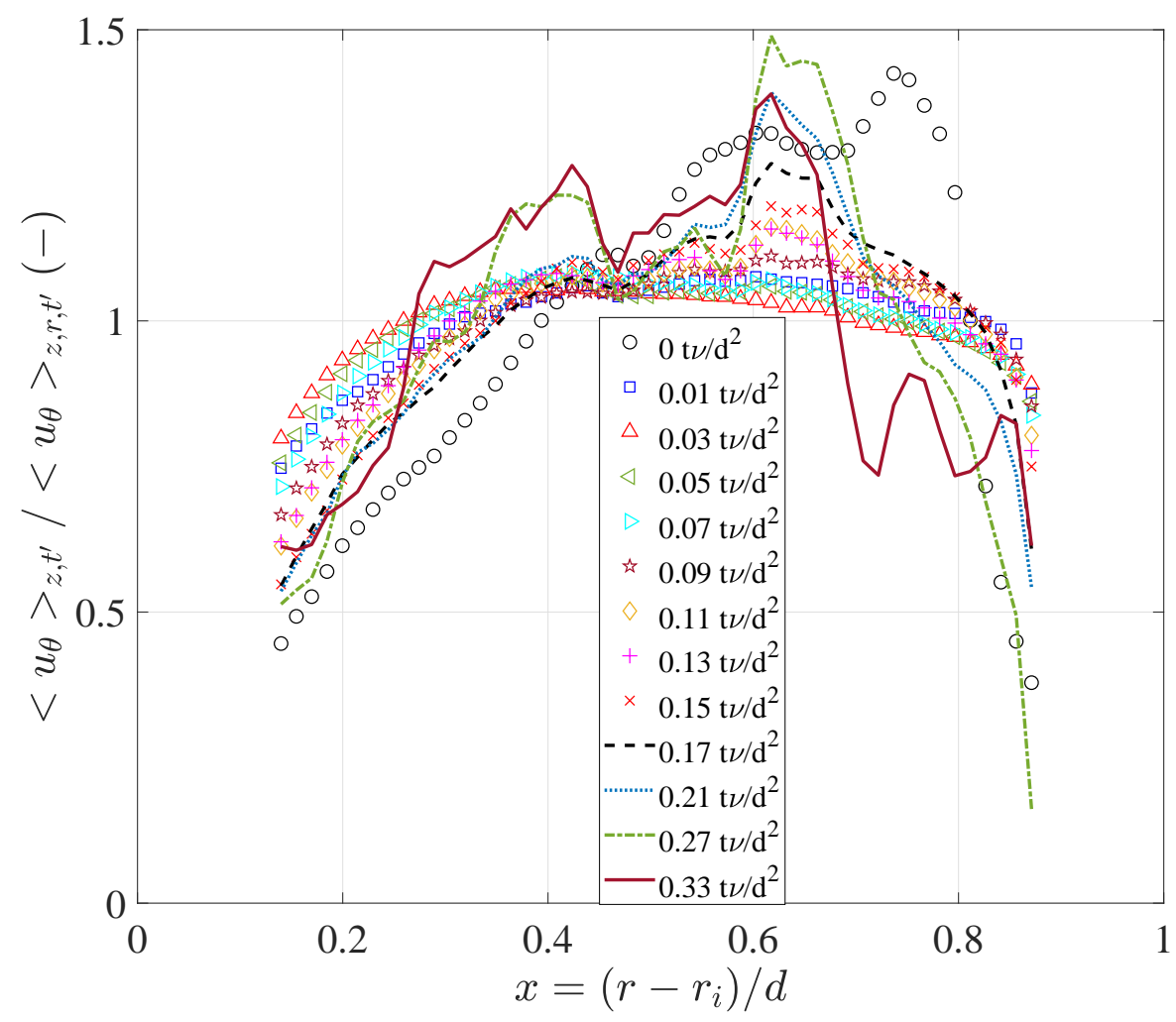

Figure 10: Radial profile of azimuthal velocity averaged axially and over time, for $t^{\prime}=\left(i-5 f d / u_{\theta}\right):\left(i+5 f d / u_{\theta}\right)$, and normalised with its spatial mean value at $R e_{o}=17300$. The time represents the instant at which the profile was extracted from the moment decay started.

This was not feasible in the current geometry due to technical constraints. Otherwise, a numerical approach can definitely shed some light on this subject as demonstrated by Ostilla-Mónico et al. (2017) and Kaiser et al. (2020).The numerical studies can provide results in the whole domain, especially in the boundary layer region and wall friction, and geometrical configurations can be modified much more easily.

\section{Conclusion}

In this study, a visualization and stereo-PIV analysis is presented for the instantaneous stoppage of the outer cylinder of a TC system which leads to the generation and consequently decay of turbulence due to the absence of any further driving force. The lowest $R e_{o}$ at which this phenomenon could be observed was found to be $R e_{o} \approx 870$. The primary criterion to observe it was the presence of laminar flow prior to the abrupt stoppage. The generation and decay of turbulence process could be described by three phases. The stoppage induces the formation of a centrifugally unstable flow region near the outer cylinder which in turn leads to the formation of axisymmetric vortices. As the velocity always decreases during the whole decay process, the growth of the vortices is accompanied by continuous readjustments as they propagate towards the inner cylinder. This induces the transition to turbulence which induces the growth of the turbulent 
and wind KE. This is the phase I also observed by Kaiser which is not present in free turbulence decay experiments as in the case of Verschoof et al. (2016) and Ostilla-Mónico et al. (2017).

Phase II starts after the maximum is reached for the wind and turbulent KE, and the production term. At this time the whole gap is filled with turbulence. The reorganization of the structures and the length scales continues. We found power law decay rate for wind KE for this phase II. The decay exponent is closer to the Kolmogorov theoretical constant of 10/7. Moreover, this phase could be represented by the Taylor microscale which increased as the power function of time but not continuously due to the reorganization of the structures. Additionally, during this phase self-similarity was observed based on the criteria presented by Verschoof et al. (2016), and also based on certain characteristics of self-similarity presented by George (1992).

The phase III corresponds to the last stage of the decay process when the decay is principally due to viscous diffusion. The final phase started when the biggest structures formed from the reorganization process starts decaying. It remains to be seen, how this decay process will unfold at much higher $R e_{o}$. An experimental analysis will be most welcoming but a numerical approach can definitely present some new ideas.

Declaration of Interests. The authors report no conflict of interest.

Funding. We would like to offer our sincere gratitude for financial support from the RIN project Tomo-PIV (grant R2018-RIN-0019) of the Normandy region.

\section{REFERENCES}

Antonia, R. A., Duenidi, L., Danaila, L. \& Tang, S. L. 2017 Small scale turbulence and the finite Reynolds number effect. Phys. Fluids 29, 020715.

Batchelor, G. K., Townsend, A. \& TAYlor, G. I. 1948a Decay of isotropic turbulence in the initial period. Proc. Roy. Soc. Lon. Ser. A. Math. and Phy. Sci. 193, 539-598.

Batchelor, G. K., Townsend, A. \& TAylor, G. I. $1948 b$ Decay of turbulence in the final period. Proc. Roy. Soc. Lon. Ser. A. Math. and Phy. Sci. 194, 527-543.

Biferale, L., Boffeta, G., Celani, A., Lanotte, A., Toschi, F. \& Vergassola, M. 2003 The decay of homogeneous anisotropic turbulence. Phys. Fluids 15, 2105-2112.

Davidson, P. A. 2011 The minimum energy decay rate in quasi-isotropic grid turbulence. Phys. Fluids 23, 085108.

Esser, A. \& Grossmann, S. 1996 Analytic expression for Taylor-couette stability boundary. Phys. Fluids 8, 1814.

Euteneuer, A. G. \& KARLSRUHe, B. R. D. 1972 Die entwieklung von längswirbeln in zeitlieh anwaehsenden grenzsehichten an konkaven wänden. Acta Mechanica 13, 215-223.

Eyink, G. L. \& Thomson, D. J. 2000 Free decay of turbulence and breakdown of self-similarity. Phys. Fluids 51, 477-479.

George, W. K. 1992 The decay of homogeneous isotropic turbulence. Phys. Fluids A 4, 1492-1509.

Huisman, S. G., van der Veen, R. C. A., Sun, C. \& Lohse, D.. 2014 Multiple states in highly turbulent Taylor couette flow. Nat. Commun. 5, 3820.

Hurst, D. \& VAssilicos, J. C. 2007 Scalings and decay of fractal-generated turbulence. Phys. Fluids 19, 035103.

Hussain, A. K. M. F. \& Reynolds, W. C. 1970 The mechanics of an organized wave in turbulent shear flow. J. Fluid Mech. 41, 241-258.

Kaiser, F., Frohnapfel, B., Ostilla-Monico, R., Kriegseis, J., Rival, D. E. \& Gatti, D. 2020 On the stages of vortex decay in an impulsively stopped, rotating cylinder. J. Fluid Mech. 885, A6 1-31.

de Karman, T. \& Howarth, L. 1938 On the statistical theory of isotropic turbulence. Phys. Lett. A 164, 192-215.

Kıм, М. С. \& Сног, С. К. 2004 The onset of Taylor-Görtler vortices in impulsively decelerating swirl flow. Korean J. Chem. Eng. 21, 767-772.

Kıм, М. С. \& Сног, С. K. 2006 The onset of Taylor-Görtler vortices during impulsive spin-down to rest. Chem. Eng. Sci. 61, 6478-6485. 
KIM, M. C., SonG, K. H. \& CHOI, C. K. 2008 Energy stability analysis for impulsively decelerating swirl flows. Phys. Fluids 20, 064101.

Kohuth, K. R. \& Neitzel, G. P. 1988 Experiments on the instability of an impulsively-initiated circular couette flow. Exp. Fluids 6, 199-208.

Kolmogorov, A. N. 1941 On degeneration (decay) of isotropic turbulence in incompressible viscous liquid. Dokl. Akad. Nauk SSSR 31, 538-540.

Lohse, D. 1994 Crossover from high to low Reynolds number turbulence. Phys. Rev. Lett. 24, 3223-3226.

Mathis, D.M. \& Neitzel, G. P. 1985 Experiments on impulsive spin-down to rest. Phys. Fluids 28, 449-454.

McСomb, W. D., Berera, A., Yoffe, S. R. \& Linkmann, M. 2014 Energy transfer and dissipation in forced isotropic turbulence. Phys. Rev. E 91, 043013.

MсСомв, W. D. \& FaIRHURST, R. B. 2018 The dimensionless dissipation rate and the Kolmogorov (1941) hypothesis of local stationarity in freely decaying isotropic turbulence. J. Mathematical Phys. 59, 073103.

Meldi, M., Sagaut, P. \& Lucor, D. 2011 A stochastic view of isotropic turbulence decay. J. Fluid Mech. 668, 351-362.

Neitzel, G. P. 1982 Marginal stability of impulsively initiated couette flow and spin-decay. Phys. Fluids 25, 226-232.

Neitzel, G. P. \& Davis, S. H. 1981 Centrifugal instabilities during spin-down to rest in finite cylinders. numerical experiments. J. Fluid Mech. 102, 329-352.

Ostilla-Mónico, R., Zhu, X., Spandan, V., Verzicco, R. \& Lohse, D. 2017 Life stages of wall-bounded decay of Taylor-couette turbulence. Phys. Rev. Fluids. 2, 114601.

Peixinho, J. \& Mullin, T. 2006 Decay of turbulence in pipe flow. Phys. Rev. Lett. 96, 094501.

Pope, Stephen B. 2000 Turbulent Flows. Cambridge University Press.

RaYleigh, O. M. 1917 On the dynamics of revolving fluids. Proc. Roy. Soc. Lon. Ser. A. Math. and Phy. Sci. 93, 148-154.

SAFFMAN, P. G. 1967 The large-scale structure of homogeneous turbulence. J. Fluid Mech. 27, 581-593.

SAVAS, Ö. 1992 Spin-down to rest in a cylindrical cavity. J. Fluid. Mech. 234, 529-552.

SchiKARSKI, T. \& Avila, M. 2017 T mixer a novel system to investigate decaying turbulence in a wallbounded environment. In 16th Eur. Turb. Conf..

SCHNEIDER, K. \& FARGE, M. 2008 Final states of decaying 2d turbulence in bounded domains: Influence of the geometry. Physica D: Nonlin. Phen. 237, 2228-2233.

Singh, H. 2016 Particle image velocimetry and computational fluid dynamics applied to study the effect of hydrodynamics forces on animal cells cultivated in Taylor vortex bioreactor. Ph.D. thesis, Universidade Federal do São Carlor - UFSCar.

Singh, H., FlETCHER, D. F. \& NiJdAM, J. J. 2011 An assessment of different turbulence models for predicting flow in a baffled tank stirred with a Rushton turbine. Chem. Eng. Sci. 66, 5976-5988.

Sinhuber, M., Bodenschatz, E. \& Bewley, G. P. 2015 Decay of turbulence at high Reynolds numbers. Phys. Rev. Lett. 114, 034501.

Skrbek, L. \& Stalp, S. R. 2000 On the decay of homogeneous isotropic turbulence. Phys. Fluids 12, 1997-2019.

Sreenivasan, K. R. 1984 On the scaling of the turbulence energy dissipation rate. Phys. Fluids 27, 10481059.

TaYlor, G. I. 1935 Statistical theory of turbulence. Proc. Roy. Soc. Lon. Ser. A. Math. and Phy. Sci. 151, 421-444.

Thormann, A. \& Maneveau, C. 2014 Decay of homogeneous, nearly isotropic turbulence behind active fractal grids. Phys. Fluids 26, 025112.

Tillmann, W. 1967 Development of turbulence during the build-up of a boundary layer at a concave wall. Phys. Fluids 10, S108.

Touil, H., Bertoglio, J.-P. \& Shao, L. 2002 The decay of turbulence in a bounded domain. Les méthodes nouvelles de la mécanique céleste $\mathbf{3}, \mathrm{N} 49$.

VAlente, P. C. \& VAssilicos, J. C. 2015 The energy cascade in grid-generated non-equilibrium decaying turbulence. Phys. Fluids 27, 045103.

Vassilicos, J. C. 2015 Dissipation in turbulent flows. Annu. Rev. of Fluid Mech. 47, 95-114.

Verschoof, R. A., Huisman, S. G., van der Veen, R. C. A., Sun, C. \& Lohse, D. 2016 Self-similar decay of high Reynolds number Taylor-couette turbulence. Phys. Rev. Fluids 1, 062402. 